\title{
Conformal Symmetries of Regions
}

\author{
ALAN F. BEARDON ${ }^{\dagger}$ AND IAN SHORT ${ }^{\dagger \dagger}$
}

\begin{abstract}
We discuss which groups arise as the group of conformal symmetries of a plane region. We also show how to compute the symmetry group of a given region.
\end{abstract}

\section{Conformal Symmetry Groups}

A conformal symmetry of a region $D$ in the complex plane is a oneto-one conformal map of $D$ onto itself. In this expository paper we discuss conformal symmetry groups, mostly for plane regions, but also for certain regions in higher dimensional spaces. In particular, we address the following two questions.

Question 1. Which groups arise as conformal symmetry groups?

Question 2. Given a region, what is its conformal symmetry group?

Let us consider Question 2 in a concrete example. Let $D$ be the region exterior to the three circles $C_{1}=\{z:|z|=16\}, C_{2}=\{z$ : $|z-(-16+24 i)|=4\}$ and $C_{3}=\left\{z:\left|z-\left(9+\frac{89}{4} i\right)\right|=t\right\}, 0<t<8$ (this region is illustrated in Figure 1). The condition $0<t<8$ ensures that none of the circles intersect; $C_{3}$ is tangent to $C_{1}$ for $t=8.0013 \cdots$. For each $0<t<8$, what is the conformal symmetry group of $D$ ? This group is determined up to isomorphism in $\S 5$, and explicitly in $\S 6$.

Let us briefly review the basic ideas regarding conformal symmetries. As our examples will have symmetries that are Möbius maps we begin by discussing Möbius maps. The extended complex plane

2000 Mathematics Subject Classification. Primary 30C20, 51B10.

$\dagger$ This paper contains further developments of the material given in this author's lecture at the DIAS Winter Symposium, 15 December 2005.

$\dagger \dagger$ Supported by Science Foundation Ireland grant 05/RFP/MAT0003. 


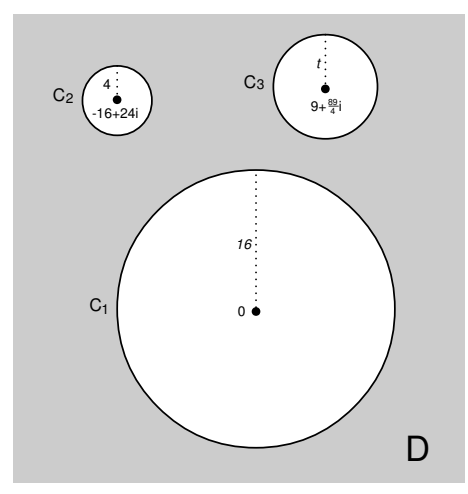

Figure 1 . The region $D$ is exterior to $C_{1}, C_{2}$ and $C_{3}$

is the union of the complex plane $\mathbb{C}$ and the point $\infty$ at infinity, and is denoted by $\mathbb{C}_{\infty}$. It is homeomorphic to the unit sphere in $\mathbb{R}^{3}$ via stereographic projection, and it acquires the structure of a Riemann surface with the introduction of the chart $z \mapsto 1 / z$ at $\infty$. The conformal automorphisms (that is, the holomorphic bijections) of $\mathbb{C}_{\infty}$ onto itself are the Möbius transformations $z \mapsto(a z+b) /(c z+d)$, where $a d-b c \neq 0$. Each Möbius transformation maps circles to circles, where a circle in $\mathbb{C}_{\infty}$ is either a Euclidean circle or a Euclidean line with $\infty$ attached. The Möbius transformations that fix $\infty$ are the maps $z \mapsto a z+b$, where $a \neq 0$. These maps form the group of conformal Euclidean similarities, and the subgroup of maps with $|a|=1$ is the group of conformal Euclidean isometries. The anti-conformal Euclidean isometries are the maps $z \mapsto a \bar{z}+b$ with $|a|=1$; more generally, the anti-conformal Möbius maps are the maps $z \mapsto(a \bar{z}+b) /(c \bar{z}+d)$, where $a d-b c \neq 0$.

A region is a non-empty, open, connected subset of $\mathbb{C}_{\infty}$. The connectivity of a region is the cardinality of the set of components of its (compact) complement, and this may be finite or infinite. Generally speaking, much is known about regions of finite connectivity; little is known about regions of infinite connectivity.

A conformal symmetry (or automorphism) of a region $D$ is a bijective holomorphic map of $D$ onto itself. In this paper a conformal symmetry is injective so that we are excluding such maps as $z \mapsto z^{2}$ which is conformal (in the sense of angle-preserving), but not injective, on $\mathbb{C} \backslash\{0\}$. Because each conformal symmetry of $D$ is a bijection, 
these symmetries form a group which we denote by $\mathrm{Aut}^{+}(D)$. An automorphism of a region that fixes three distinct points is the identity map (see [8] and [10]). Also, it is known that if $D$ is a region of finite connectivity which is at least three, then any holomorphic proper self-map of $D$ is a conformal symmetry of $D$ (see [11]).

We end this introduction with a simple example to show that there are regions whose conformal symmetry group is trivial. In fact, the region we construct has a much stronger property: it has no holomorphic self-maps other than the identity map. Let $E=\{1,-1, i, \zeta, \infty\}$, where $\zeta=(1+i) / \sqrt{2}$, and let $f$ be a non-constant holomorphic map of $D=\mathbb{C}_{\infty} \backslash E$ into itself. Picard's Theorem implies that each point of $E$ is a removable singularity of $f$, so that $f$ extends to a function that is meromorphic on $\mathbb{C}_{\infty}$ and hence is a rational map. As $f$ maps $D$ into itself we see that $f^{-1}(E) \subset E$, and as $f$ is rational (and therefore surjective) we conclude that $f(E)=E=f^{-1}(E)$. It follows that $f$ is a bijection of $E$ onto itself, and so some iterate, say $f^{k}$ of $f$, acts as the identity on $E$. In particular, $f^{k}(z)=\infty$ if and only if $z=\infty$, so that $f^{k}$ is a polynomial, say of degree $d$. As $f(z)=1$ if and only if $z=1$, and similarly for -1 , there must be constants $A$ and $B$ such that $A(z-1)^{d}+1=f(z)=B(z+1)^{d}-1$. This can only happen if $d=1$; thus $f^{k}$, and hence $f$ also, is a Möbius map. As $f$ is Möbius, it preserves the concyclic nature of any four points. The circles in $\mathbb{C}_{\infty}$ that contain $\infty$ are the Euclidean lines, and each Euclidean line meets $E$ in at most three points (one of which is $\infty$ ); therefore $f$ must map the set $\{1,-1, i, \zeta\}$ of four concyclic points onto itself. We deduce that $f$ must fix $\infty$, so that $f$ is a Euclidean similarity. Clearly, the only Euclidean similarity that maps $\{1,-1, i, \zeta\}$ onto itself is the identity map. For more examples of this type, see [3].

\section{Reduction to a Problem in Geometry}

In 1851 Riemann gave what is now known as the Riemann Mapping Theorem: each simply connected proper subregion of $\mathbb{C}$ is conformally equivalent to the open unit disc $\mathbb{D}$. For a discussion of the history of this result, see [12, page 181]. Koebe provided a rigorous proof of Riemann's theorem and, in 1920, he also proved a much stronger result, namely that each finitely connected region is conformally equivalent to a circular region, where a circular region is a region whose complement is a union of mutually disjoint closed discs. 
In 1993, this result was extended to countably connected regions by He and Schramm [6], who also showed that the conformal symmetries of circular regions of countable connectivity are Möbius maps (this was known previously for circular regions of finite connectivity). Although it is not known whether an arbitrary region $D$ is conformally equivalent to a circular region, Maskit [10] has shown that there is a region $D^{\prime}$ conformally equivalent to $D$ such that $\mathrm{Aut}^{+}\left(D^{\prime}\right)$ consists only of Möbius transformations. In this paper we answer questions 1 and 2 only for circular regions of finite connectivity such that each closed disc in the complement of the region has positive radius. For regions whose complement has closed discs of zero radius, see [3].

Each conformal Möbius transformation acting on $\mathbb{C}_{\infty}$ is a composition of an even number of inversions in circles in $\mathbb{C}_{\infty}$, and each anti-conformal Möbius transformation is a composition of an odd number of inversions. We embed $\mathbb{C}$ as the plane $x_{3}=0$ in $\mathbb{R}^{3}$. If we regard a circle in $\mathbb{C}_{\infty}$ as the 'equator' of a sphere in $\mathbb{R}^{3}$, we see that each Möbius transformation can be extended (as the same composition of inversions) so as to act on $\mathbb{R}^{3} \cup\{\infty\}$ in such a way that it preserves the upper-half $\mathbb{H}^{3}=\left\{\left(x_{1}, x_{2}, x_{3}\right) \mid x_{3}>0\right\}$ of $\mathbb{R}^{3}$. Now, any real Möbius transformations that preserves the upper-half $\mathbb{H}^{2}=\left\{\left(x_{1}, x_{2}\right) \mid x_{2}>0\right\}$ of the complex plane acts as an isometry of the hyperbolic plane $\mathbb{H}^{2}$ with the hyperbolic metric $d s^{2}=\left(d x_{1}^{2}+d x_{2}^{2}\right) / x_{2}^{2}$. In a similar way, any complex Möbius transformation acts on $\mathbb{H}^{3}$, which we regard as a model of hyperbolic 3 -space with metric $d s^{2}=\left(d x_{1}^{2}+d x_{2}^{2}+d x_{3}^{2}\right) / x_{3}^{2}$. In fact, the group of (conformal and anti-conformal) Möbius transformations is precisely the group of isometries of hyperbolic space $\mathbb{H}^{3}$ (see [1] for details). A corollary of this important fact is that complex analytic questions about conformal symmetries of circular regions can be reduced to geometric questions about the action of the Möbius group of isometries of hyperbolic space $\mathbb{H}^{3}$. Each circle in $\mathbb{C}_{\infty}$ is the ideal boundary of a hemisphere in $\mathbb{H}^{3}$, and these hemispheres are the hyperbolic planes in $\mathbb{H}^{3}$ (just as semicircles in $\mathbb{H}^{2}$ orthogonal to $\mathbb{R}$ are geodesics).

We illustrate these ideas in Figure 2. The circular region $D$ lying in $\mathbb{C}_{\infty}$ is bounded by four circles. One of these circles is $C$, and this is the ideal boundary of the hyperbolic plane $\Pi$. The region enclosed by the four hyperbolic planes is an unbounded hyperbolic polyhedron, $P$, which has ideal boundary $\bar{D}$. In addition, we can 

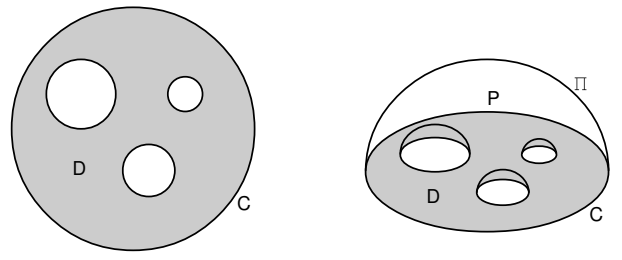

FiguRE 2

think of $P$ as the hyperbolic convex hull of $D$; this is the union of all hyperbolic geodesics whose endpoints lie in $D$.

\section{The Solution to Question 1}

In general, a conformal symmetry of a region $D$ is defined only on $D$ and it need not extend analytically beyond (or even to) the boundary of $D$. However, the conformal symmetries of a circular region of countable connectivity are Möbius transformations and these are defined on the whole of $\mathbb{C}_{\infty}$. This is an important point, and this extra information can be used to answer Question 1 in the case of regions of finite connectivity. First, any region of finite connectivity is, by Koebe's result, conformally equivalent to a circular region of finite connectivity. Obviously, conformally equivalent regions have isomorphic symmetry groups so in answering Question 1, we may confine our attention to circular regions of finite connectivity.

Suppose that $D=\mathbb{C}_{\infty} \backslash\left(E_{1} \cup \cdots \cup E_{m}\right)$, where $E_{1}, \ldots, E_{m}$ are disjoint closed discs and $m \geqslant 3$. Each conformal symmetry of $D$, necessarily a Möbius transformation, permutes these discs. This argument provides a homomorphism

$$
\Phi: \operatorname{Aut}^{+}(D) \rightarrow S_{m}, \quad f \mapsto \Phi_{f},
$$

where $\Phi_{f}(i)=j$ if and only if $f\left(E_{i}\right)=E_{j}$, and where $S_{m}$ is the permutation group on $m$ symbols. If $f$ lies in the kernel of $\Phi$ then $f$ fixes each $E_{i}$ set-wise. By the Brouwer Fixed Point Theorem, $f$ has a fixed point in each $E_{i}$. Thus $f$ has at least three fixed points and so is the identity map. Thus $\Phi$ is injective, and hence $\operatorname{Aut}^{+}(D)$ is, up to isomorphism, a subgroup of $S_{m}$.

It is well known that each finite Möbius group is isomorphic either to the cyclic or dihedral symmetry group of a 2-dimensional regular polygon or to the rotational symmetry group of a 3 -dimensional 
regular polyhedron (a Platonic solid). See either [1, $\S 5.1]$ or $[7$, Chapter VI] for a proof, although we sketch a proof later in this section. There are exactly five Platonic solids: the tetrahedron, cube, octahedron, dodecahedron and icosahedron, and their rotational symmetry groups are, in order, $A_{4}, S_{4}, S_{4}, A_{5}$ and $A_{5}$. The cube and octahedron have the same rotational symmetry group as they are dual polyhedra; the dodecahedron and icosahedron are also dual polyhedra. These facts show that each finite Möbius group is either cyclic, dihedral or isomorphic to one of $A_{4}, S_{4}$ or $A_{5}$. All such groups arise as conformal symmetry groups, and we have now answered Question 1 completely.

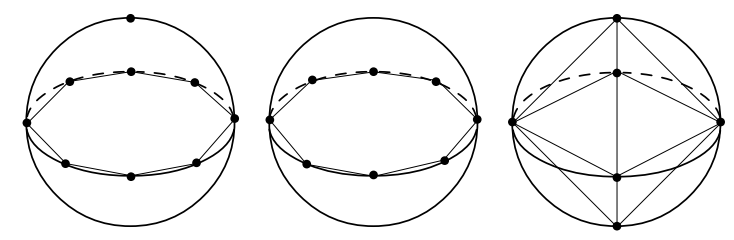

FiguRE 3

We illustrate these ideas in Figure 3, where each sphere has a number of points removed. The resulting regions have conformal symmetry groups, from left to right, $C_{8}, D_{8}$ and $A_{4}$. Two octagons and an octahedron are shown connecting the points to indicate the relationship between subgroups of the Möbius group and the symmetries of the polygons and polyhedra.

Let us sketch a proof that each finite Möbius group $G$ is isomorphic to a finite group of rotations of $\mathbb{R}^{3}$. Each $g$ in $G$ acts on hyperbolic space $\mathbb{H}^{3}$. As $G$ is finite, each orbit in $\mathbb{H}^{3}$ is a finite set. Take any one orbit, say $\mathcal{O}$, and enclose it in the smallest possible closed hyperbolic ball $B$ in $\mathbb{H}^{3}$, and let $\zeta$ be the hyperbolic centre of $B$. This construction is possible because $\mathcal{O}$ is finite. As $\mathcal{O}$ is $G$-invariant, so is $B$, and hence so is $\zeta$. We can now map $\mathbb{H}^{3}$ bijectively onto the open unit ball $\mathbb{B}^{3}$ in $\mathbb{R}^{3}$ (by a Möbius map acting on $\left.\mathbb{R}^{3} \cup\{\infty\}\right)$ with $\zeta$ mapping to the origin. The elements of $G$ transform into Euclidean rotations, so $G$ is isomorphic (indeed, conjugate in the larger Möbius group) to a finite group of Euclidean rotations of $\mathbb{R}^{3}$. 
We conclude this section with a brief discussion of the higher dimensional case. A (conformal or anti-conformal) Möbius map acting on $\mathbb{R}^{m} \cup\{\infty\}$ is a composition of inversions across $(m-1)$ dimensional spheres or hyperplanes, and Liouville proved that any conformal or anti-conformal map acting on a subdomain of $\mathbb{R}^{m} \cup\{\infty\}$ is Möbius. In answer to Question 2 in this case, for each finite group $G$ there is a circular region in some $\mathbb{R}^{m} \cup\{\infty\}$ such that the group of conformal symmetries of $D$ is isomorphic to $G$.

\section{The Inversive Distance}

In general, to understand symmetry groups it is useful to identify group invariants. The inversive distance $\chi\left(C_{1}, C_{2}\right)$ between two Euclidean circles $C_{1}$ and $C_{2}$ in $\mathbb{C}$ is given by the equation

$$
\chi\left(C_{1}, C_{2}\right)=\left|\frac{\left|c_{1}-c_{2}\right|^{2}-r_{1}^{2}-r_{2}^{2}}{2 r_{1} r_{2}}\right|,
$$

where $C_{1}$ has centre $c_{1}$ and radius $r_{1}$, and $C_{2}$ has centre $c_{2}$ and radius $r_{2}$. There is a similar formula when one or both of $C_{1}$ and $C_{2}$ are Euclidean lines. The inversive distance is invariant under Möbius transformations. If $C_{1}$ and $C_{2}$ are disjoint then there is a Möbius transformation $f$ which maps $C_{1}$ and $C_{2}$ to concentric circles of radii $s_{1}$ and $s_{2}$ that are centred on the origin, in which case $\chi\left(C_{1}, C_{2}\right)=\frac{1}{2}\left(s_{1} / s_{2}+s_{2} / s_{1}\right)$.

The inversive distance has a simple interpretation with hyperbolic geometry. The circles $C_{1}$ and $C_{2}$ are the ideal boundaries of hyperbolic planes $\Pi_{1}$ and $\Pi_{2}$ in $\mathbb{H}^{3}$. If $C_{1}$ and $C_{2}$ are disjoint then $\Pi_{1}$ and $\Pi_{2}$ are separated by a positive hyperbolic distance $\rho$ (see Figure 4), and $\chi\left(C_{1}, C_{2}\right)=\cosh \rho$. If $C_{1}$ and $C_{2}$ intersect in an angle $\theta$ in $[0, \pi / 2]$ then $\chi\left(C_{1}, C_{2}\right)=\cos \theta$. For more information about the inversive distance, see [1] and [5, page 129].

We can use this invariant of Möbius transformations to calculate conformal symmetry groups. Consider a circular region $D$ with boundary circles $C_{1}, \ldots, C_{m}$, each of positive radius, and corresponding hyperbolic planes $\Pi_{1}, \ldots, \Pi_{m}$. If $\phi \in \operatorname{Aut}^{+}(D)$ then

$$
\chi\left(\phi\left(C_{i}\right), \phi\left(C_{j}\right)\right)=\chi\left(C_{i}, C_{j}\right) \quad(i, j=1, \ldots, m) .
$$

A converse to this observation, which is discussed in the next section, enables us to answer Question 2. 


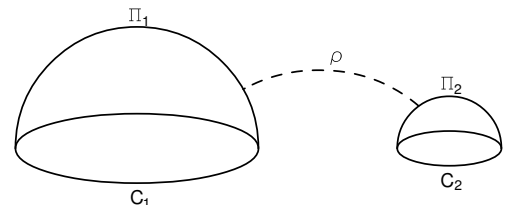

FiguRE 4

\section{The Solution to Question 2}

Let $\Omega$ and $\Omega^{\prime}$ be two circular regions in $\mathbb{C}_{\infty}$ bounded by circles $C_{j}$ and $C_{j}^{\prime}, j=1, \ldots, m$, each of positive radius.

Theorem 5.1. There exists a hyperbolic isometry $\phi$ with $\phi(\Omega)=\Omega^{\prime}$ and $\phi\left(C_{j}\right)=C_{j}^{\prime}$ for each $j=1, \ldots, m$ if and only if $\chi\left(C_{j}, C_{k}\right)=$ $\chi\left(C_{j}^{\prime}, C_{k}^{\prime}\right)$ for all $j, k=1, \ldots, m$.

We emphasise that the hyperbolic isometry $\phi$ in Theorem 5.1 may correspond to a conformal or an anti-conformal Möbius transformation.

The circles $C_{j}$ in Theorem 5.1 are pairwise disjoint. The theorem can be generalised to allow some, but not all, collections of circles. An example of the failure of Theorem 5.1 when circles are allowed to intersect is shown in Figure 5 . The equalities $\chi\left(C_{i}, C_{j}\right)=\chi\left(C_{i}^{\prime}, C_{j}^{\prime}\right)$ hold for $i, j=1,2,3,4$; each $\chi\left(C_{i}, C_{j}\right)$ is either 0 or 1 depending on whether $C_{i}$ and $C_{j}$ are orthogonal or parallel. The regions $D$ and $D^{\prime}$ are conformally equivalent as they are both simply connected. Nevertheless, there is not a conformal map $\phi: D \rightarrow D^{\prime}$ with $\phi\left(C_{i}\right)=$ $C_{i}^{\prime}$ for $i=1,2,3,4$, because the rectangular regions are not similar, in the Euclidean sense.
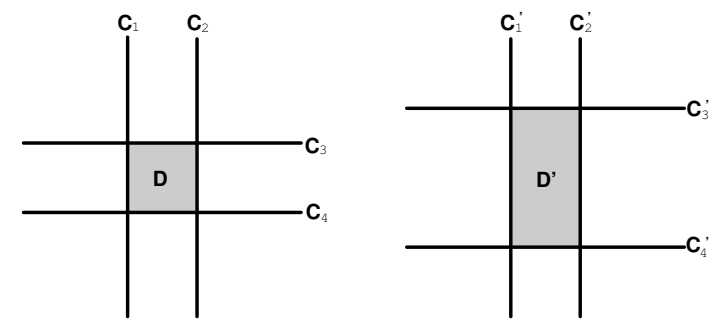

FiguRE 5 
A proof of Theorem 5.1 is discussed in the next section.

Question 2 can now be answered with the help of Theorem 5.1. The existence of a conformal symmetry is equivalent to the equality of certain inversive distances, and these inversive distances are easily computed using (4.1). Consider the example in Figure 1. We have

$$
\begin{array}{r}
\chi\left(C_{1}, C_{2}\right)=\frac{35}{8}, \quad \chi\left(C_{1}, C_{3}\right)=\frac{5121-16 t^{2}}{512 t} \text { and } \\
\chi\left(C_{2}, C_{3}\right)=\frac{9793-256 t^{2}}{128 t} .
\end{array}
$$

It can be checked that $\chi\left(C_{2}, C_{3}\right)>\chi\left(C_{1}, C_{3}\right)$ and $\chi\left(C_{2}, C_{3}\right)>$ $\chi\left(C_{1}, C_{2}\right)$ for each $t \in(0,8)$. Also, $\chi\left(C_{1}, C_{3}\right)=\chi\left(C_{1}, C_{2}\right)$ if and only if $t=9 / 4$. Therefore when $t=9 / 4$ there is a Möbius map $\sigma$ (possibly anti-conformal) that fixes $C_{1}$ and interchanges $C_{2}$ and $C_{3}$. In fact, $f$ is conformal therefore $\mathrm{Aut}^{+}(D)$ is isomorphic to $C_{2}$ when $t$ equals $9 / 4$, and otherwise $\operatorname{Aut}^{+}(D)$ is trivial. We show this in $\S 6$.

\section{Reduction to a Problem in Linear Algebra}

There are several models of hyperbolic space and, for calculating symmetries of circular regions (or, equivalently, for calculating hyperbolic isometries that preserve a particular collection of hyperbolic planes), there are many advantages to the hyperboloid model. To construct the hyperboloid model of 3-dimensional hyperbolic space, we endow $\mathbb{R}^{4}$ with the Lorentz inner-product

$$
\langle x, y\rangle=x_{1} y_{1}+x_{2} y_{2}+x_{3} y_{3}-x_{4} y_{4} .
$$

The hyperboloid sheet

$$
\mathcal{H}=\left\{x \in \mathbb{R}^{4}:\langle x, x\rangle=-1, x_{4}>0\right\}
$$

becomes a model of 3 -dimensional hyperbolic space with metric $d$ determined by

$$
\cosh d(x, y)=-\langle x, y\rangle .
$$

A hyperbolic plane $\Pi$ in $\mathcal{H}$ is the intersection of $\mathcal{H}$ with a 3 -dimensional Euclidean plane $P$ through the origin. Each such plane $P$ has a Lorentz unit normal $n$ such that $P=\{x:\langle x, n\rangle=0\}$. The hyperbolic isometries of $\mathcal{H}$ are the positive Lorentz orthogonal maps, namely the linear isomorphisms of $\mathbb{R}^{4}$ that preserve the Lorentz inner-product and map $\mathcal{H}$ to $\mathcal{H}$. A hyperbolic isometry $\phi$ maps one hyperbolic plane $\Pi$ to another, $\Pi^{\prime}$, if and only if $\phi$ maps a 
unit normal $n$ of $\Pi$ to a unit normal $n^{\prime}$ of $\Pi^{\prime}$. The inversive distance between $\Pi$ and $\Pi^{\prime}$ is $\left|\left\langle n, n^{\prime}\right\rangle\right|$.

The elements of a proof of Theorem 5.1 are all present in the hyperboloid model. A collection of hyperbolic planes can be represented by associated unit normals $n_{1}, \ldots, n_{m}$, and the inversive distances are $\left|\left\langle n_{i}, n_{j}\right\rangle\right|$, for $i, j=1, \ldots, m$. Let $n_{j}$ and $n_{j}^{\prime}$ be unit normals associated to $C_{j}$ and $C_{j}^{\prime}$ in Theorem 5.1. By taking some (or all) of the Lorentz normals to the planes as basis vectors, one can construct a linear transformation $\phi$ that maps $n_{j}$ to $n_{j}^{\prime}$ for each $j$, and the Lorentz orthogonality of $\phi$ is ensured by the inversive distance condition.

There are other benefits of the hyperboloid model. The proof of Theorem 5.1 is valid for more general collections of boundary circles $C_{j}$; in particular, there may be infinitely many circles. The Lorentz model of hyperbolic space can be constructed in all dimensions, and likewise Theorem 5.1 generalises to all dimensions. Also, the proof of Theorem 5.1 can be adapted to give results analogous to Theorem 5.1 about punctured spheres.

The final advantage of the hyperboloid model that we mention is that it allows one to construct conformal symmetry groups explicitly, not just up to isomorphism. Consider the example of Figure 1. In $\S 5$ we showed that the automorphism group is trivial unless $t=9 / 4$, in which case the automorphism group is $C_{2}$. One can choose Lorentz normals $n_{1}, n_{2}$ and $n_{3}$ in $\mathbb{R}^{4}$ that correspond to the circles $C_{1}, C_{2}$ and $C_{3}$ and then construct a Lorentz orthogonal map $L$ that fixes $n_{1}$ and interchanges $n_{2}$ and $n_{3}$. The Möbius map $\sigma$ corresponding to $L$ is conformal because $\operatorname{det} L=1$ (the alternative is that if $\operatorname{det} L=-1$ then $\sigma$ is anti-conformal). In this case, $\operatorname{Aut}^{+}(D)=\{1, \sigma\}$,

$$
L=\left(\begin{array}{cccc}
-1 & 0 & 0 & 0 \\
0 & \frac{-41}{9} & 0 & \frac{-40}{9} \\
0 & \frac{425}{12} & \frac{257}{32} & \frac{3485}{96} \\
0 & \frac{1285}{36} & \frac{255}{32} & \frac{10537}{288}
\end{array}\right)
$$

and

$$
\sigma(z)=\frac{20 i z+256}{z-20 i} .
$$

(One can check that the Lorentz orthogonal map $L$ and the Möbius map $\sigma$ correspond to the same hyperbolic isometry using $[14$, Theorem 10].) 


\section{Regions of Infinite ConneCtivity}

Questions 1 and 2 are more complicated for regions of infinite connectivity, even for regions with a countable number of boundary components. There are many examples of such regions and their automorphisms groups in the literature. For a simple example, $\mathrm{Aut}^{+}(\mathbb{C} \backslash \mathbb{Z})$ is infinite, and is generated by $z \mapsto-z$ and $z \mapsto z+1$. More complicated examples arise in the theory of discrete Möbius groups where the groups known as Schottky groups play a prominent role. Without going into details here, one can construct many examples of a perfect, Cantor-like set $L$ (the limit set), and discrete, finitely or infinitely generated, free groups of Möbius conformal symmetries of $\mathbb{C}_{\infty} \backslash E$.

We shall content ourselves here by discussing just one example (for an extension of these ideas see [2]). Let $D$ be the complement of a sequence $z_{1}, z_{2}, \ldots$ of distinct points in $\mathbb{C}$ that converges to $\infty$. Next, let $f$ be a non-constant holomorphic self-map of $D$ ( $f$ may be, but need not be, a conformal symmetry of $D$, and it need not be injective). By Picard's Theorem, each $z_{n}$ is a removable singularity for $f$, and it is easy to see that for each $n, f\left(z_{n}\right) \neq \infty$. Thus $f$ extends to an entire function. As $f$ maps $D$ into itself, so too do all the iterates of $f$, and Montel's Theorem (on three omitted values) implies that the family of iterates is normal in $D$. Thus the Julia set of $f$ lies in $\left\{z_{1}, z_{2}, \ldots, \infty\right\}$. It is well known that the Julia set of an entire function is uncountable unless the function is a linear polynomial (see [4]). We conclude that the entire function $f$ is a linear polynomial; thus every holomorphic self-map of $D$ is of the form $z \mapsto a z+b$, where $a \neq 0$. In particular, the group of conformal symmetries of $D$ is a group of Mobius maps. Each (Möbius) conformal symmetry of $D$ must fix $\infty$. Moreover, $\mathrm{Aut}^{+}(D)$ cannot contain any maps $g(z)=a z+b$ with $|a| \neq 1$ as otherwise the set of images of $z$ under the forward and backward iterates of $g$ accumulate at the finite fixed point of $g$. Therefore $\mathrm{Aut}^{+}(D)$ consists only of Euclidean rotations and translations. As $E \cap \mathbb{C}$ is discrete, and invariant under the conformal symmetries of $D, \mathrm{Aut}^{+}(D)$ must be a discrete group of Euclidean isometries. Thus, as is well known, $\mathrm{Aut}^{+}(D)$ is either a finite cyclic group of Euclidean rotations, or one of the three conformal frieze groups (these are the discrete Euclidean isometry groups whose translations form a cyclic subgroup), or one of the five conformal wallpaper groups (whose translations form an 
abelian subgroup of rank two). Of course, in the problem we are discussing here the determination of $\mathrm{Aut}^{+}(D)$ depends on the precise location of the points $z_{n}$. In fact, for almost all choices of the points $z_{n}$, the group $\operatorname{Aut}^{+}(D)$ is trivial.

\section{REFERENCES}

[1] A. F. Beardon, The Geometry of Discrete Groups, Graduate Texts in Mathematics, 91, Springer-Verlag, 1983.

[2] A. F. Beardon, Holomorphic self maps of a region, preprint.

[3] A. F. Beardon and D. Minda, Conformal automorphisms of finitely connected plane regions, to appear.

[4] W. Bergweiler, Iteration of meromorphic functions, Bull. Amer. Math. Soc. (2) 29 (1993), 151-188.

[5] H. S. M. Coxeter and S. L. Greitzer, Geometry Revisited, Math. Association of America, 1967.

[6] Z-X. He and O. Schramm, Fixed points, Koebe uniformization and circle packings, Ann. of Math. 137 (1993), 369-406.

[7] L. R. Ford, Automorphic functions, Amer. Math. Soc., second edition, 1972.

[8] K. Leschinger, Über Fixpunkte holomorpher Automorphismen, Manuscripta Math. (4) 25 (1978), 391-396.

[9] R. C. Lyndon, Groups and Geometry, London Math. Soc. Lecture Notes, 101, Cambridge University Press, 1985.

[10] B. Maskit, The conformal group of a plane domain, Amer. J. Math. 90 (1968), 718-722.

[11] C. Mueller and W. Rudin, Proper holomorphic self-maps of plane regions, Complex Variables Theory Appl. (1-2) 17 (1991), 113-121.

[12] R. Remmert, Classical Topics in Complex Function Theory, Graduate Texts in Mathematics, 172, Springer-Verlag, 1998.

[13] I. Short, Conformal symmetries of spherical regions, preprint.

[14] J. B. Wilker, Inversive geometry, The Geometric Vein, Ed. Davis, C., Grünbaum, B. and Sherk, F. A., Springer-Verlag, 1981, 379-442.

Alan F. Beardon, Department of Pure Mathematics and Mathematical Statistics, Centre for Mathematical Sciences, University of Cambridge,

Wilberforce Road, Cambridge CB3 0WB, United Kingdom

a.f.beardon@dpmms.cam.ac.uk
Ian Short,

Mathematics Department, Logic House, NUI Maynooth, Maynooth, Co. Kildare, Ireland ian.short@nuim.ie

Received on 25 November 2006. 